\title{
Aging and the effects of a half marathon on Achilles tendon force-elongation relationship
}

Citation for published version (APA):

Ackermans, T. M. A., Epro, G., McCrum, C., Oberlaender, K. D., Suhr, F., Drost, M. R., Meijer, K., \& Karamanidis, K. (2016). Aging and the effects of a half marathon on Achilles tendon force-elongation relationship. European Journal of Applied Physiology, 116(11-12), 2281-2292.

https://doi.org/10.1007/s00421-016-3482-z

Document status and date:

Published: 01/12/2016

DOI:

10.1007/s00421-016-3482-z

Document Version:

Publisher's PDF, also known as Version of record

Document license:

Taverne

Please check the document version of this publication:

- A submitted manuscript is the version of the article upon submission and before peer-review. There can be important differences between the submitted version and the official published version of record.

People interested in the research are advised to contact the author for the final version of the publication, or visit the DOI to the publisher's website.

- The final author version and the galley proof are versions of the publication after peer review.

- The final published version features the final layout of the paper including the volume, issue and page numbers.

Link to publication

\footnotetext{
General rights rights.

- You may freely distribute the URL identifying the publication in the public portal. please follow below link for the End User Agreement:

www.umlib.nl/taverne-license

Take down policy

If you believe that this document breaches copyright please contact us at:

repository@maastrichtuniversity.nl

providing details and we will investigate your claim.
}

Copyright and moral rights for the publications made accessible in the public portal are retained by the authors and/or other copyright owners and it is a condition of accessing publications that users recognise and abide by the legal requirements associated with these

- Users may download and print one copy of any publication from the public portal for the purpose of private study or research.

- You may not further distribute the material or use it for any profit-making activity or commercial gain

If the publication is distributed under the terms of Article $25 \mathrm{fa}$ of the Dutch Copyright Act, indicated by the "Taverne" license above, 


\title{
Aging and the effects of a half marathon on Achilles tendon force-elongation relationship
}

\author{
Thijs Maria Anne Ackermans ${ }^{1,2}$ - Gaspar Epro ${ }^{1,3}$ - Christopher McCrum ${ }^{1,2}$. \\ Kai Daniel Oberländer ${ }^{4}$ Frank Suhr ${ }^{5} \cdot$ Maarten Robert Drost $^{2} \cdot$ Kenneth Meijer $^{2}$. \\ Kiros Karamanidis $^{1}$
}

Received: 21 April 2016 / Accepted: 25 September 2016 / Published online: 30 September 2016

(C) Springer-Verlag Berlin Heidelberg 2016

\begin{abstract}
Purpose We aimed to determine whether there are different changes in Achilles tendon (AT) mechanical properties in middle-aged, compared to younger runners that might indicate that tendon fatigue, induced by long-distance running, is age-dependent.

Methods 27 middle-aged (50-67 years) and 22 younger (21-29 years) participants ran a $21 \mathrm{~km}$ route at their own pace (mean and SD: old: $3.1 \pm 0.3 \mathrm{~m} \mathrm{~s}^{-1}$; young: $\left.3.6 \pm 0.5 \mathrm{~m} \mathrm{~s}^{-1}\right)$. We tested for changes in the AT force-elongation relationship using dynamometry and
\end{abstract}

Communicated by Olivier Seynnes.

Electronic supplementary material The online version of this article (doi:10.1007/s00421-016-3482-z) contains supplementary material, which is available to authorized users.

Kiros Karamanidis

karamanidis@dshs-koeln.de

1 Institute of Movement and Sport Gerontology, German Sport University Cologne, Am Sportpark Müngersdorf 6, 50933 Cologne, Germany

2 Department of Human Movement Science, NUTRIM School of Nutrition and Translational Research in Metabolism, Maastricht University Medical Centre+, Universiteitssingel 60, 6229 ER Maastricht, The Netherlands

3 Institute of Biomechanics and Orthopaedics, German Sport University Cologne, Am Sportpark Müngersdorf 6, 50933 Cologne, Germany

4 Fresenius University of Applied Sciences, Media School, Im Mediapark 4C, 50670 Cologne, Germany

5 Department of Molecular and Cellular Sport Medicine, Institute of Cardiovascular Research and Sport Medicine, German Sport University Cologne, Am Sportpark Müngersdorf 6, 50933 Cologne, Germany ultrasonography during isometric voluntary ankle plantarflexion ramp contractions, conducted $20-28 \mathrm{~h}$ pre-run, immediately pre-run, immediately post-run and $20-28 \mathrm{~h}$ post-run. Stride frequency and number were examined to estimate cyclic tensile loading characteristics of the tendon during running.

Results Muscle strength decreased significantly $(P<0.05)$ in both groups immediately post-run (old: $17 \%$; young: $11 \%$ ) and recovered to baseline within $20-28 \mathrm{~h}$ post-run. AT stiffness did not change for the younger adults, whereas the middle-aged adults showed a significant $(P<0.05)$ decrease in AT stiffness (22\%). However, tendon stiffness recovered to baseline $20-28 \mathrm{~h}$ post-run. Middle-aged, compared to young adults, demonstrated significantly $(P<0.05)$ greater stride frequency and number, but no correlations with tendon fatigue changes were determined $\left(R^{2} \leq 0.038\right)$.

Conclusions The results suggest that the plasticity of the AT in response to short-term mechanical loading may be age dependent and that the AT length-tension properties of middle-aged runners may be more vulnerable to change following running compared to younger athletes. However, the observed AT changes in the middle-aged runners dissipated within $20-28 \mathrm{~h}$ post-run, suggesting that a tendon viscoelastic recovery mechanism may occur in vivo.

Keywords Tendon stiffness - Tendon fatigue $\cdot$ Running · Mechanical loading $\cdot$ Age $\cdot$ Muscle contraction
Abbreviations
1dayPre-Run
20-28 $\mathrm{h}$ before the run
1dayPost-Run
AT
Within 20-28 $\mathrm{h}$ after the run
ANOVA
Achilles tendon
CSA
Analysis of variance
Cross-sectional area 


$\begin{array}{ll}\text { GM } & \text { Gastrocnemius medialis } \\ \text { imPre-Run } & \begin{array}{l}\text { Immediately before the run } \\ \text { imPost-Run }\end{array} \\ \text { Immediately after the run } \\ \text { MTU } & \begin{array}{l}\text { Muscle-tendon unit } \\ \text { MVC }\end{array} \\ & \begin{array}{l}\text { Maximal voluntary plantarflexion ramp } \\ \text { contraction }\end{array} \\ \text { SD } & \text { Standard deviation }\end{array}$

\section{Introduction}

Tendons play a crucial role in movement by transmitting muscle forces to the skeleton and, therefore, changes in human tendon biomechanical properties could affect movement effectiveness (Ker et al. 1987; Biewener and Roberts 2000; Karamanidis and Arampatzis 2005; Lichtwark et al. 2007) and injury risk (Lavagnino et al. 2014). This may be of particular concern for older populations, who experience age-related changes in tendon composition that may affect the mechanical properties of the tendon which could eventually reduce the tendon's tolerance of mechanical loading.

Despite a lack of clarity in the literature, most in vitro studies in humans point toward the fact that aging can be associated with decreased tensile stiffness and ultimate failure strength of tendons and collagenous tissues (Noyes and Grood 1976; Tkaczuk 1968; Viidik 1982). In a more recent study by Lavagnino et al. (2014), using both in vitro animal and in vivo human experiments, it was shown that tendon composition and material properties diminish the ability of elongated tendons to re-establish normal tension homeostasis, making an older tendon more prone to overuse injuries after repetitive cyclic loading exercise (Lavagnino et al. 2014). This may be one explanation that older age has been identified as a predisposing factor for overuse injuries (Huttunen et al. 2014; Jozsa et al. 1989; Kvist 1994), as well as longer recovery times following tendon repair procedures (Lavagnino et al. 2013, 2014). Specifically, Achilles tendon (AT) injuries appear to be age related, as the incidence rate of AT injury in men over 40 years is almost twice as high as in men between 18 and 39 years of age (Huttunen et al. 2014). An additional risk factor for AT injury appears to be activities with repetitive cyclic mechanical loading, such as long-distance running, as runners are one of the most susceptible populations to chronic AT injuries and AT ruptures (Brunet et al. 1990; Clement et al. 1984). During running, the AT has to cope with multiple tensile strain cycles, consisting of high strain rates and magnitudes (e.g., AT strain magnitude up to $5.5 \%$; Lichtwark et al. 2007). Such loading profiles can lead to the tendon becoming more compliant and more susceptible to large strains, which can ultimately lead to tendon failure (Ker et al. 2000; Wang et al. 1995). These changes in the tendon force-elongation relationship are suggested to be caused by a disequilibrium between the rate of damage and the rate of repair (Ker et al. 2000), due to significant changes in the expression of collagens (downregulation of Col I and upregulation of Col III and Col V), fiber angulation (increase in density of the fibers) and delamination among adjacent fibers (separation of the fibers into layers; Fung et al. 2010). Such changes in the tendon force-elongation relationship, morphology and molecular profile due to cyclic mechanical loading are collectively termed tendon fatigue (Wang et al. 1995; Fung et al. 2010; Ker et al. 2000), and the response of tendons to in vitro and in vivo cyclic mechanical loading has been well established in animal models (Fung et al. 2010; Lavagnino et al. 2014; Wang et al. 1995). Improved understanding of potential age-related differences in the response of the AT to long-distance running might be important for reducing the risk of AT injuries in middle- and older-aged runners.

In previous in vivo human studies, various dynamic activities (such as heel drop exercise, running or hopping) have been used to examine whether changes in tendon mechanical properties occurred that might be indicative of tendon fatigue, following repetitive cyclic loading (Farris et al. 2012; Lichtwark et al. 2013; Obst et al. 2016; Peltonen et al. 2010, 2012). However, no significant change in the AT force-elongation relationship in vivo has been found in three (Farris et al. 2012; Peltonen et al. 2010, 2012) of these five studies. The studies of Lichtwark et al. (2013) and Obst et al. (2016) did find an increase in strain at a given force, which could be indicative of early stage mechanical fatigue. However, these effects were much smaller than those predicted by both ex vivo and in vivo animal experiments (Fung et al. 2010; Lavagnino et al. 2014; Wang et al. 1995). This may have been due to the methodology or the nature of the loading profiles used in these studies. Crucially, these studies were conducted with predominantly young subjects (mean age under 30 years; Farris et al. 2012; Lichtwark et al. 2013; Peltonen et al. 2010). As a consequence, there is limited evidence for how the human AT in middle-aged to older runners (50 years and older) responds to a long-lasting cyclic loading exercise such as long-distance running. To our knowledge, one study (Mademli and Arampatzis 2008) investigated the AT fatigue response in older adults in vivo during cyclic submaximal isometric voluntary contractions on a dynamometer, but failed to find any significant age-related fatigue effects on the mechanical properties of the AT. However, the contraction protocol used may not have simulated the typical mechanical loading profile of a long-distance run. This evidence gap is significant because older aged runners are at a higher risk for AT injury (Kvist 1994) and the finding that aging diminishes tendon fatigue quality during extended repetitive cyclic loading in in vitro and in vivo animal models (Lavagnino et al. 2014). 
The purpose of this study was, therefore, to examine whether there are different changes in AT force-elongation properties between middle-aged and younger adult longdistance runners following a half marathon run that might indicate that tendon fatigue, in response to repetitive cyclic loading, is age dependent. We decided to use a half marathon run $(20.5 \mathrm{~km})$ at a self-selected pace, as this includes a high number of loading cycles and represents a typical training and competition distance for long-distance runners. The number and frequency of strides were assessed to indicate the absolute amount and frequency of AT loading cycles experienced by the runners. It was hypothesized that the AT would exhibit greater mechanical changes in the middle-aged compared to the younger adult long-distance runners, which would be consistent with tendon fatigue. Specifically, we expected that the AT would exhibit a more pronounced decrease in tendon stiffness for the middleaged, compared to the younger adult long-distance runners.

\section{Methods}

\section{Subjects}

The investigation was conducted with 27 middle-aged [age: $56 \pm 4.7$ years; height: $180.4 \pm 5.9 \mathrm{~cm}$; body mass: $74.6 \pm 6.1 \mathrm{~kg}$; mean and standard deviation (SD)] and 22 younger (age: $26 \pm 2.6$ years; height: $182.1 \pm 6.7 \mathrm{~cm}$; body mass: $74.7 \pm 9.0 \mathrm{~kg}$ ) male recreational marathon runners. The subjects had been regular runners for at least the last 4 and 6 years, for the younger and middle-aged adults, respectively. All subjects participated in competitive longdistance races (a minimum of two races within the last 2 years) and had a minimum training volume of $30 \mathrm{~km}$ per week (average of about $50 \mathrm{~km}$ per week across subjects). Exclusion criteria were any previous AT ruptures, AT injury (e.g., tendinopathy) or pain within the last 12 months, or musculoskeletal impairments in the lower limbs (e.g., ankle joint pain) determined by interview and questionnaire that could influence the findings of the study. The study was approved by the ethical board of the German Sport University Cologne, the procedures of the study were explained to the participants and written informed consent was obtained from all individual participants included in the study prior to the testing in accordance with the Declaration of Helsinki.

\section{Experimental design}

All subjects performed a half marathon run $(20.5 \mathrm{~km})$ on a flat, off-road track. The subjects were asked to run the course as fast as possible at their self-selected pace and they were accompanied and encouraged by one of the staff members, who cycled alongside them. The mechanical properties of the triceps surae muscle-tendon unit (MTU) of the dominant leg (preferred leg for step initiation; determined by a questionnaire) were assessed on four occasions: (a) 20-28 h before the run (1dayPre-Run), (b) immediately before the run (imPre-Run), (c) immediately after the run (imPost-Run) and (d) within 20-28 h after the run (1dayPost-Run). The two pre-measurements (1dayPre-Run and imPre-Run) were used to test the day-to-day reliability in muscle strength and tendon stiffness. The measurement 1dayPost-Run was performed to investigate if the potential tendon changes were still present 1 day after the run. The stride number and frequency of the individuals were examined, to investigate if potential changes in the AT forceelongation relationship were affected by average AT cyclic loading frequency and total number of AT loading cycles. All subjects were instructed not to perform any additional physical exercise (e.g., running) during these 3 days of measurements.

\section{Analysis of the tendon force-elongation relationship and muscle strength}

The mechanical properties of the triceps surae MTU of the dominant leg were assessed by integrating ultrasonography (MyLab One, Esaote; Genova, Italy) and dynamometry synchronously using a custom-made device (Fig. 1). The subjects were seated with their lower leg secured and with their foot on a custom-made strain gauge-type dynamometer (sampling frequency $1000 \mathrm{~Hz}$ ) with the ankle and knee joints positioned at $90^{\circ}$ (thigh and foot perpendicular to the shank; see supporting data for this setup in Online Resource 1). A laser-guided electrical potentiometer system was used to position the subject's foot on the force plate by setting the midpoint of the malleolus lateralis in line with the force plate's center of rotation (Fig. 1c). The position of the seat relative to the dynamometer was recorded by two additional electrical potentiometers, to reliably replicate the positions during each of the four MTU measurements (the height of the seat, distance of the seat to the force plate and position of the foot on the force plate; as shown in Fig. 1c).

To examine muscle strength and the force-elongation relationship of the tendon during the loading phase, all subjects performed four isometric plantarflexion contractions at different force levels: a maximal voluntary plantarflexion ramp contraction (MVC), followed by three sustained contractions at 30,50 and $80 \%$ of the maximal joint moment determined during the MVC. At every measurement session, a new MVC was performed, and force levels at 30, 50 and $80 \%$ of the maximal joint moment (calculated from the new MVC) were used. All sustained contractions were guided by visual feedback of the joint moment produced by the subject on a screen. The resultant ankle joint 
(A)

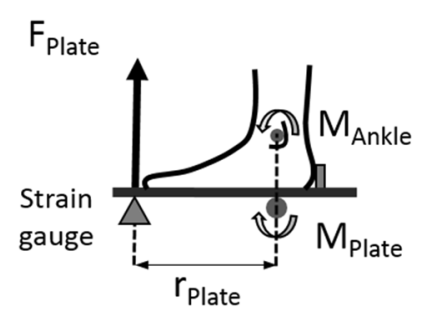

(B)

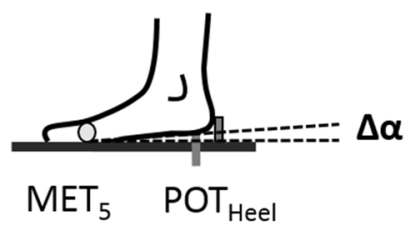

(C)

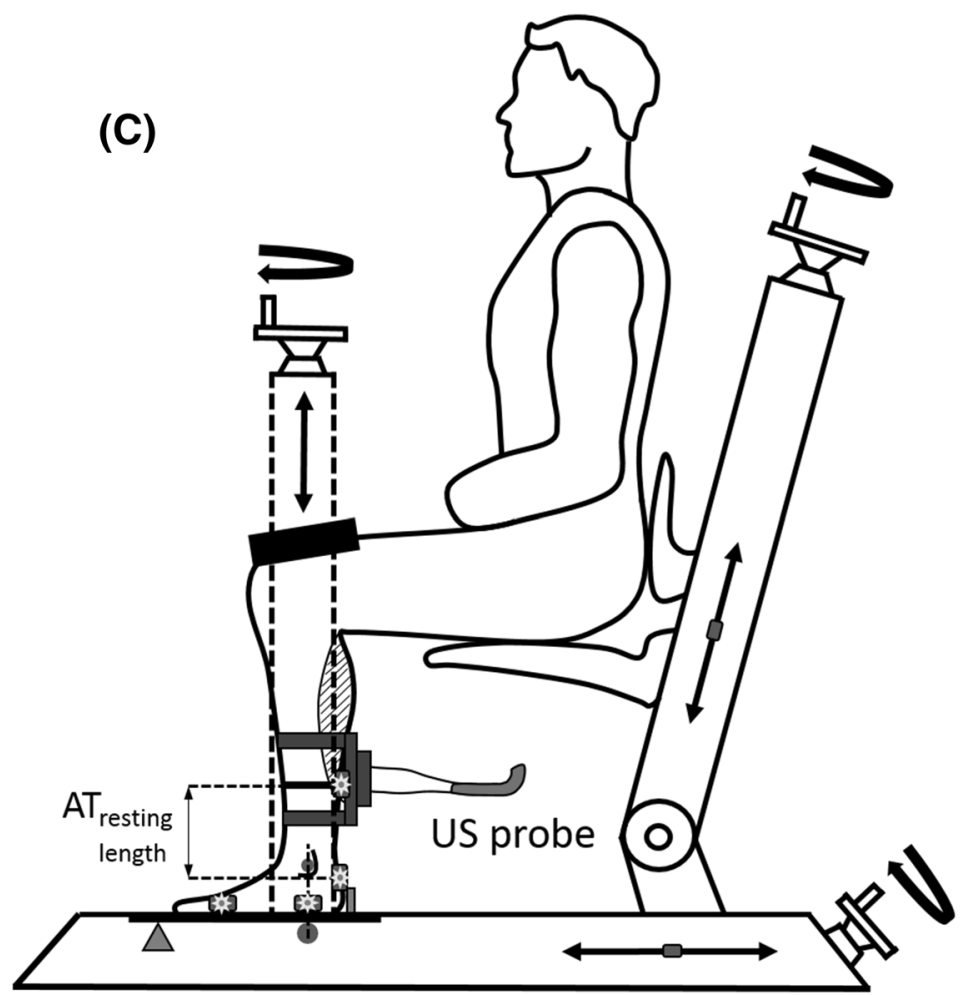

Fig. 1 Diagram of the used custom-made strain gauge-type dynamometer. The subjects were seated with their lower leg secured and their foot on the dynamometer, with the ankle and knee joints positioned at $90^{\circ}(\mathbf{c})$. A laser-guided potentiometer system was used to position the midpoint of the malleolus lateralis in line with the force plate's center of rotation and to calculate the tendon's resting length (ATresting length) between the most proximal point of the tuber calcanei and an adhesive tape placed on the skin close to the GM myotendinous junction. The position of the seat relative to the dynamometer (horizontal and vertical position) was recorded by

moment was calculated using inverse dynamics. Due to aligning the axis of rotation of the ankle in line with the force plate's center of rotation, the ankle joint moment could be considered equal to the moment of the force plate (Fig. 1a). Adjustment of moments due to gravitational and compression forces was carried out for all subjects before each plantarflexion contraction. However, it is important to note that the resultant ankle joint moment is an approximation of the moment produced by the triceps surae, as it does not account for the moment contributions of the other synergistic agonist muscles or the antagonist dorsiflexors. For the calculation of the AT force, the resultant ankle joint moment was divided by the tendon moment arm obtained from the literature (Maganaris et al. 1998). Prior to the measurements (except imPost-Run) the subject performed ten submaximal and three maximal cyclic loading contractions to precondition the tendon (Maganaris 2003). No tendon preconditioning was done immediately after the run (imPost-Run), since this was assumed to be unnecessary two additional potentiometers. The axis of rotation of the ankle was aligned with the force plate's center of rotation; therefore, the ankle joint moment (MAnkle) was equal to the moment of the force plate (MPlate), which is the product of FPlate (resultant force of the force plate) and rPlate (moment arm of the force plate; a). The ankle joint changes $(\Delta \alpha)$ during the contractions were calculated via the inverse tangent of the ratio of the heel lift (calculated by potentiometer POTHeel) to the distance between MET5 (position of the head of fifth metatarsal bone) and POTHeel (b)

after a $21 \mathrm{~km}$ run with a great number of mechanical loading cycles.

Elongation of the tendon during the loading phase was examined by visualizing the gastrocnemius medialis (GM) tendon using an ultrasound probe (sample frequency: $27 \mathrm{~Hz}$ ) fixed in the longitudinal direction at the myotendinous junction of the GM. After tendon preconditioning, the resting length of the AT was determined (individually for each measurement session) using a laser system linked to electronic potentiometers as the distance from the AT insertion to the calcaneus (identified by palpation during the first measurement 1 day pre-run) to the adhesive tape placed on the skin close to the GM myotendinous junction (Fig. 1c). The GM resting length was obtained by correcting the measured distance with the gap between the myotendinous junction and the adhesive tape (registered via electronic potentiometers), which was later used for the registration of any motion of the probe relative to the skin during the contractions (Karamanidis and Arampatzis 2005). 
The elongation of the tendon during MVC was determined by digitizing the myotendinous junction in custommade MATLAB software (MATLAB 2013b, MathWorks Inc., Natick, Massachusetts, USA). This was conducted at rest and at the three sustained (held by the subjects for $3 \mathrm{~s}$ ) contractions, which were collected at $27 \mathrm{~Hz}$ after a steady state was achieved at the predetermined target force level $(0,30,50,80 \%)$. All analog signals and ultrasound images were synchronized using a TTL signal and an input signal for the ultrasound via the EKG channel, which was specifically constructed from the manufactory in Maastricht (ESAOTE, Maastricht, The Netherlands). In the presented study, a range of $\pm 5 \%$ of the target force was accepted (when the force was held for $3 \mathrm{~s}$ ). If this was not achieved, then the specific trial was repeated. The tendon elongation at maximal force (100\%) was obtained via linear extrapolation of the values at 50 and $80 \%$ of the maximal force and the corresponding elongation of the tendon during the entire loading phase. This was determined by interpolating the data between two data points of the three sustained contractions (using linear fits between two points which surrounded the given tendon force level) for all subjects. The effect of potential ankle joint angular rotation on the measured elongation of the tendon during contraction was taken into account using the product of the ankle joint angle changes and the Achilles tendon moment arm taken from Maganaris et al. (1998). In the current study, we used a potentiometer located under the heel to measure any heel lift during contraction and, thereby, calculated the changes in the ankle joint angle from rest until maximal plantarflexion moment via the inverse tangent of the ratio of the heel lift to the distance between the head of the fifth metatarsal bone and the potentiometer (Fig. 1b; see also Online Resource 1). The tendon stiffness was calculated by the divided change in force by change in elongation from 30 to $80 \%$ of MVC. To account for the differences in force levels between the four measurements, the individual given tendon force at 30 and $80 \%$ of MVC was obtained from the lowest MVC from all measurements for each subject. These two values were used to calculate the stiffness for the three other measurement sessions at the corresponding force levels.

\section{Analysis of stride and velocity characteristics during running}

The stride number and frequency were assessed during running using a smartphone (Samsung Galaxy Nexus, Samsung Electronics, Seoul, South Korea) attached over the lower back at the level of the lower lumbar spine (L5) of the subjects using a semi-elastic waistband. A custom-made data acquisition application was used to save the smartphones' GPS (sample rate $1 \mathrm{~Hz}$ ) and 3-axis accelerometer
( $\pm 16 \mathrm{~g}$ range, sample rate $100 \mathrm{~Hz}$ ) data, during the run. A MATLAB program processed the recorded data and initial foot contact was identified by the minimum of the signal obtained after applying a Gaussian continuous wavelet transformation to the vertical acceleration data (method adapted from McCamley et al. (2012)). Based on the above procedure, we calculated for each individual the stride frequency and total amount of strides, as well as the average and maximal (calculated over an interval of $1 \mathrm{~km}$ ) running velocity during the half marathon exercise. The parameters were acquired to get an indication about individual running patterns and, hence, AT loading characteristics.

\section{Statistics}

To examine potential differences between age groups and the different analyzed time points (1dayPre-Run, imPre-Run, imPost-Run and 1dayPost-Run) in the mechanical properties of the triceps surae MTU (tendon stiffness, tendon length changes at a given force level (every $500 \mathrm{~N}$ until a maximum of $3000 \mathrm{~N}$; performed from linear fits), maximal tendon elongation and maximal ankle joint moment), a two-factor repeated measures analysis of variance (ANOVA), with time point and age (middle-aged vs. younger; independent variable) as factors was used. For those parameters where a significant time-point effect was found, the time points imPre-Run, imPost-Run and 1dayPost-Run were calculated as a ratio to time point 1dayPre-Run and were compared between age groups and time points by a two-way repeated measures ANOVA with age as an independent factor. This was done to examine whether the magnitude of relative changes (in relation to baseline-1dayPre-Run) in the significant parameters were age dependent. When a significant time point effect or age $\times$ time point interaction was detected, a Duncan post hoc test was performed to examine where exactly the differences occurred. Subjects' age, height, mass, running data (half marathon tested time and weekly training volume), running velocity (average and maximal velocity) and stride number and frequency were investigated using an independent $t$ test to test for differences between the middle-aged and younger groups. Simple linear regression was performed to examine the effect of running velocity and stride number and frequency on the ratio of the tendon stiffness in imPost-Run to 1dayPre-run. To test the day-to-day reliability in muscle strength and tendon stiffness, we additionally calculated the intraclass correlation coefficient (ICC) between 1dayPre-Run and imPre-Run. The level of significance was set at $\alpha=0.05$. All statistical analyses were performed using Statistica (Release 7.1; Statsoft, Tulsa, OK, USA). All results in the text, tables and figures are presented as mean and SD. 
Table 1 Mean $( \pm \mathrm{SD})$ triceps surae muscle-tendon unit mechanical properties assessed at all time points for the middle-aged and younger adult long-distance runners

\begin{tabular}{|c|c|c|c|c|c|c|c|c|}
\hline & \multicolumn{4}{|c|}{ Middle-aged $(n=27)$} & \multicolumn{4}{|l|}{ Young $(n=22)$} \\
\hline & 1dayPreRun & imPreRun & imPostRun & 1dayPostRun & 1dayPreRun & imPre Run & imPostRun & 1dayPostRun \\
\hline $\begin{array}{l}\text { Joint } \\
\text { moment }_{\max } \\
\left(\mathrm{N} \mathrm{m} \mathrm{kg}^{-1}\right)^{*}\end{array}$ & $2.9(0.6)$ & $2.9(0.5)$ & $2.4^{\mathrm{a}, \mathrm{b}, \mathrm{c}}(0.7)$ & $2.8(0.6)$ & $3.5(0.6)$ & $3.6(0.5)$ & $3.1^{\mathrm{a}, \mathrm{b}, \mathrm{c}}(0.6)$ & $3.5(0.6)$ \\
\hline $\begin{array}{l}\text { Resting length } \\
(\mathrm{mm})\end{array}$ & $235.0(33.1)$ & $235.3(29.0)$ & $230.6(32.7)$ & $236.0(27.1)$ & $223.2(21.3)$ & $224.2(21.7)$ & $228.2(22.7)$ & $224.5(19.8)$ \\
\hline $\begin{array}{l}\text { Tendon elonga- } \\
\text { tion }_{\max }(\mathrm{mm})\end{array}$ & $8.9(1.9)$ & $9.2(1.9)$ & $9.0(2.2)$ & $8.9(1.9)$ & $10.1(2.2)$ & $9.9(1.9)$ & $9.8(2.0)$ & $10.0(2.1)$ \\
\hline $\begin{array}{l}\text { Tendon } \\
\text { stiffness } \\
\left(\mathrm{N} \mathrm{mm}^{-1}\right)^{*}\end{array}$ & $983.1(233.1)$ & $978.6(310.6)$ & $769.0^{\mathrm{a}, \mathrm{b}, \mathrm{c}}(298.0)$ & $940.6(284.1)$ & $1390.4(597.2)$ & $1261.1(541.6)$ & $1171.8(375.6)$ & $1331.2(475.9)$ \\
\hline
\end{tabular}

1dayPreRun 20-28 h before the half marathon run; imPreRun immediately before the half marathon run; imPostRun immediately after the half marathon run; 1dayPostRun 20-28 h after the half marathon run

* Significant age effect $(P<0.05)$

${ }^{\text {a }}$ Significant difference to 1dayPre-Run $(P<0.05)$

b Significant difference to imPre-Run $(P<0.05)$

c Significant difference to 1dayPost-Run $(P<0.05)$

\section{Results}

Regarding the muscle strength, there were statistically significant $(P<0.05)$ age and time point effects on maximal isometric ankle plantarflexion joint moments, with lower values for the middle-aged compared to the younger runners (Table 1). The post hoc analysis showed significantly $(P<0.05)$ lower maximal ankle joint moments immediately after the run (imPost-Run) in comparison to all other analyzed time points, independent of age (old and young), with comparable values between 1dayPost-Run and both pre-run measurements (Table 1). Accordingly, there was a statistically significant $(P<0.05)$ time point effect on the ratios of the maximal ankle joint moments with no age effect or age $\times$ time point interaction (Fig. 2). The ratio imPost-Run to 1dayPre-run of the maximal ankle joint moments was significantly $(P<0.05)$ lower than all other ratios, independent of age (Fig. 2). The ratios between imPre-Run to 1dayPre-Run and between 1dayPost-Run to 1dayPre-run of the maximal ankle joint moments were not significantly different. Hence, the values of the maximal ankle joint moments of both age groups came back to baseline, and the triceps surae muscle strength was recovered, 20-28 $\mathrm{h}$ after the run (Fig. 2).

A two-way ANOVA revealed a significant $(P<0.05)$ age $\times$ time point interaction on tendon stiffness (Table 1 ; Fig. 2). Similar to the muscle strength, the middle-aged runners showed significantly $(P<0.05)$ lower tendon stiffness values compared to the younger runners at all analyzed time points (Table 1). Immediately after the run (imPost-Run), tendon stiffness of the middle-aged runners

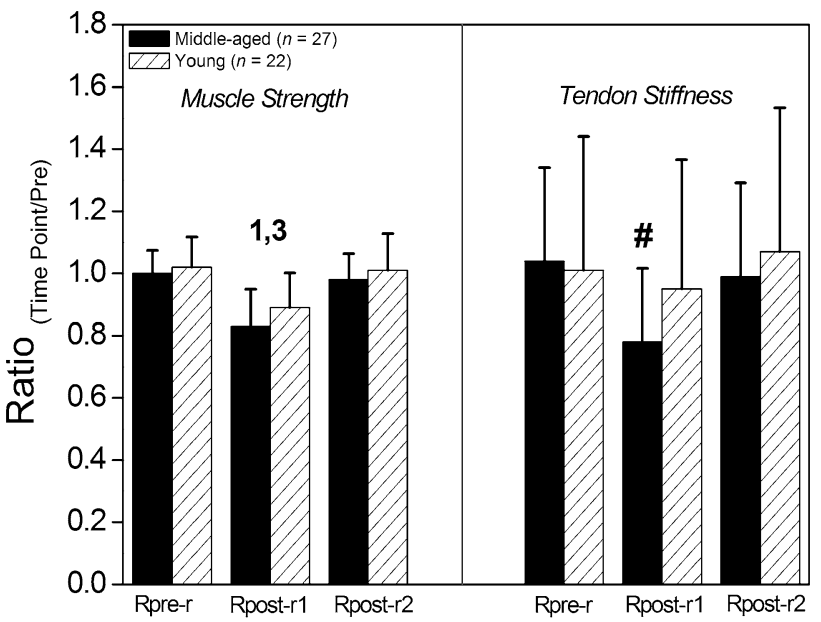

Fig. 2 Mean (and SD) ratio (the time points imPre-Run, imPost-Run and 1dayPost-Run were calculated as a ratio to time point 1dayPreRun) of the muscle strength (Joint Moment ${ }_{\max }$ ) and tendon stiffness, calculated for the different time points to the baseline measurement for the middle-aged and younger adult long-distance runners. (Rpre-r ratio immediately before the run to $20-28 \mathrm{~h}$ before the run; Rpost-rl ratio immediately after the run to 20-28 h before the run; Rpost-r2

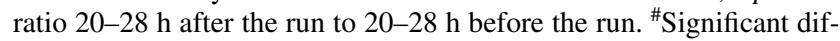
ference to all other ratios for young and old $(P<0.05)$. ${ }^{1}$ Significant difference to Rpre-r $(P<0.05)$. ${ }^{3}$ Significant difference to Rpost-r2 $(P<0.05)$

was significantly $(P<0.05)$ lower compared to both prerun measurements (1dayPre-Run and imPre-Run) and the measurement 20-28 $\mathrm{h}$ after the run (Table 1; Fig. 3). In contrast to the middle-aged runners, the younger runners 


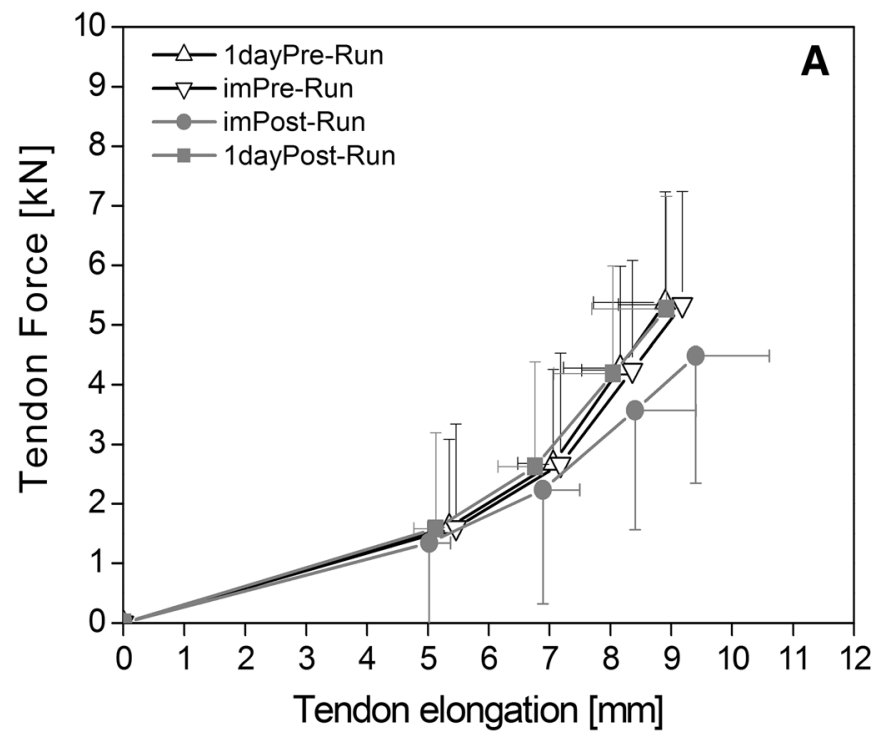

Fig. 3 Force-elongation relationship (mean and SD) of the tendon for the different time points for the middle-aged (a) and younger (b) adult long-distance runners. (1dayPre-run 20-28 h before the run;

showed no statistically significant differences in tendon stiffness post-running in comparison to all other analyzed time points (Table 1). A high day-to-day variability in the absolute stiffness values for the younger runners was seen in the pre-run measurements (difference of $129.3 \mathrm{~N} \mathrm{~mm}^{-1}$ between 1dayPre-Run and imPre-Run), but the mean of the individual ratios of tendon stiffness for the pre-run measurements showed a value close to 1.0 for both age groups (stiffness ratio for the middle aged: 1.04; stiffness ratio for the younger 1.01). Furthermore, the ratios of the tendon stiffness showed a statistically significant $(P<0.05)$ age $\times$ time point interaction, with no changes in the ratio for the young (Fig. 2). The middle-aged runners, however, showed a significantly $(P<0.05)$ lower ratio imPost-Run to 1dayPre-run compared to all other values, including the ratio imPost-Run to 1dayPre-run of the younger runners (Fig. 2). As a consequence, a significant $(P<0.05)$ relative decrease in tendon stiffness post-running was present for the middle-aged in comparison to the younger runners, but by the measurement $20-28 \mathrm{~h}$ after the run, the decrease in tendon stiffness of the middle-aged runners dissipated (1dayPre-Run; Fig. 2). Concerning our day-to-day reliability analysis in MTU mechanical properties, we found ICC values of 0.96 and 0.64 for the maximal plantarflexion moment and tendon stiffness, respectively, between 1dayPre-Run and imPre-Run.

Our comparison of the tendon elongation between groups revealed a significant $(P<0.05)$ time point effect on tendon elongation values for a given tendon force up to $2000 \mathrm{~N}$ (examined at intervals of $500 \mathrm{~N}$, Table 2). Tendons of both age groups experienced more elongation after

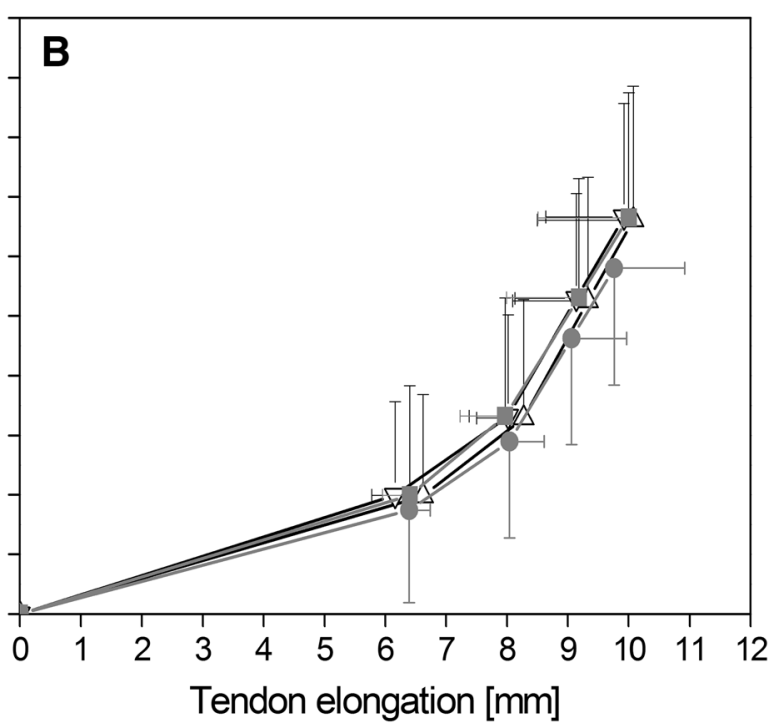

imPre-Run immediately before the run; imPost-Run immediately after the run; 1dayPost-Run 20-28 $\mathrm{h}$ after the run)

the run (imPost-Run) compared to before and returned to baseline $20-28 \mathrm{~h}$ after the run (1dayPost-Run). The force level of $2000 \mathrm{~N}$ corresponds to 29 and $37 \%$ of MVC across the younger and middle-aged participants, respectively. At the higher force levels $(2500$ and $3000 \mathrm{~N})$, there was an age $\times$ time point interaction in tendon elongation (Table 2). The middle-aged runners experienced more tendon elongation immediately after the run compared to both pre-measurements and 20-28 $\mathrm{h}$ after the run (1dayPost-Run). There was no statistically significant effect on maximal tendon elongation during MVC or resting length (Table 1).

Half marathon tested time of the middle-aged runners was, on average, $16 \%$ slower $(P<0.05)$ than the younger runners (old: $1.54 \pm 0.12 \mathrm{~h}$; young: $1.38 \pm 0.12 \mathrm{~h}$ ) and accordingly there was a significantly lower average running velocity for the middle-aged adults (old: $3.1 \pm 0.3 \mathrm{~m} \mathrm{~s}^{-1}$; young: $3.6 \pm 0.5 \mathrm{~m} \mathrm{~s}^{-1}$; Fig. 4). Furthermore, the middle-aged runners showed significantly $(P<0.05)$ higher total number of strides (old: $9977 \pm 674$ strides; young: $7890 \pm 1100$ strides $)$, increased stride frequency (old: $1.44 \pm 0.08$ strides s $^{-1}$;

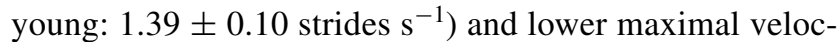
ity (old: $3.6 \pm 0.6 \mathrm{~m} \mathrm{~s}^{-1}$; young: $4.2 \pm 0.7 \mathrm{~m} \mathrm{~s}^{-1}$; Fig. 4). However, no significant effect of running velocities and stride number and frequency on the ratio of tendon stiffness imPost-Run to 1dayPre-run was found (average running velocity: $R^{2}=0.006, P=0.60$; maximal running velocity: $R^{2}=0.020, P=0.34$; total number of strides: $R^{2}=0.021, P=0.33$; stride frequency: $R^{2}=0.038$, $P=0.20$; Fig. 5). The middle-aged runners had significantly $(P<0.05)$ longer training histories (6 vs. 12 years); 
Table 2 Mean $( \pm \mathrm{SD})$ tendon elongation values assessed at different force levels during the loading phase at all time points for the middle-aged and younger adult long-distance runners

\begin{tabular}{|c|c|c|c|c|c|c|c|c|}
\hline \multirow[t]{2}{*}{ Tendon force $(\mathrm{N})$} & \multicolumn{4}{|c|}{ Middle-aged $(n=27)$} & \multicolumn{4}{|c|}{ Young $(n=22)$} \\
\hline & 1dayPreRun & imPreRun & imPost Run & 1dayPost Run & 1dayPreRun & imPre Run & imPost Run & 1dayPostRun \\
\hline 500 & $1.7(0.54)$ & $1.7(0.50)$ & $1.9^{\mathrm{a}, \mathrm{b}, \mathrm{c}}(0.57)$ & $1.6(0.47)$ & $1.7(0.42)$ & $1.6(0.47)$ & $1.9^{\mathrm{b}, \mathrm{c}}(0.50)$ & $1.6(0.34)$ \\
\hline 1000 & 3.4 (1.07) & $3.4(0.99)$ & $3.8^{\mathrm{a}, \mathrm{b}, \mathrm{c}}(1.10)$ & $3.3(0.93)$ & $3.4(0.84)$ & $3.2(0.95)$ & $3.7^{\mathrm{b}, \mathrm{c}}(1.00)$ & $3.2(0.67)$ \\
\hline 1500 & $4.9(1.35)$ & $5.0(1.44)$ & $5.3^{\mathrm{a}, \mathrm{b}, \mathrm{c}}(1.35)$ & $4.8(1.20)$ & $5.1(1.26)$ & $4.7(1.40)$ & $5.5^{\mathrm{b}, \mathrm{c}}(1.41)$ & $4.8(0.97)$ \\
\hline 2000 & $6.0(1.42)$ & $6.1(1.62)$ & $6.5^{\mathrm{a}, \mathrm{b}, \mathrm{c}}(1.52)$ & $5.9(1.30)$ & $6.3(1.47)$ & $6.0(1.59)$ & $6.8^{\mathrm{b}, \mathrm{c}}(1.56)$ & $6.1(1.22)$ \\
\hline 2500 & $6.7(1.34)$ & $6.8(1.60)$ & $7.2^{\mathrm{a}, \mathrm{b}, \mathrm{c}}(1.59)$ & $6.5(1.27)$ & $7.2(1.70)$ & $6.8(1.61)$ & $7.5(1.53)$ & $7.0(1.46)$ \\
\hline 3000 & $7.3(1.50)$ & $7.4(1.66)$ & $7.8^{\mathrm{a}, \mathrm{b}, \mathrm{c}}(1.65)$ & $7.1(1.43)$ & $7.8(1.82)$ & $7.5(1.59)$ & $8.0(1.57)$ & $7.6(1.54)$ \\
\hline
\end{tabular}

1dayPreRun 20-28 h before the half marathon run; imPreRun immediately before the half marathon run; imPostRun immediately after the half marathon run; 1dayPostRun $20-28 \mathrm{~h}$ after the half marathon run

a Significant difference to 1dayPre-Run $(P<0.05)$

b Significant difference to imPre-Run $(P<0.05)$

c Significant difference to 1dayPost-Run $(P<0.05)$
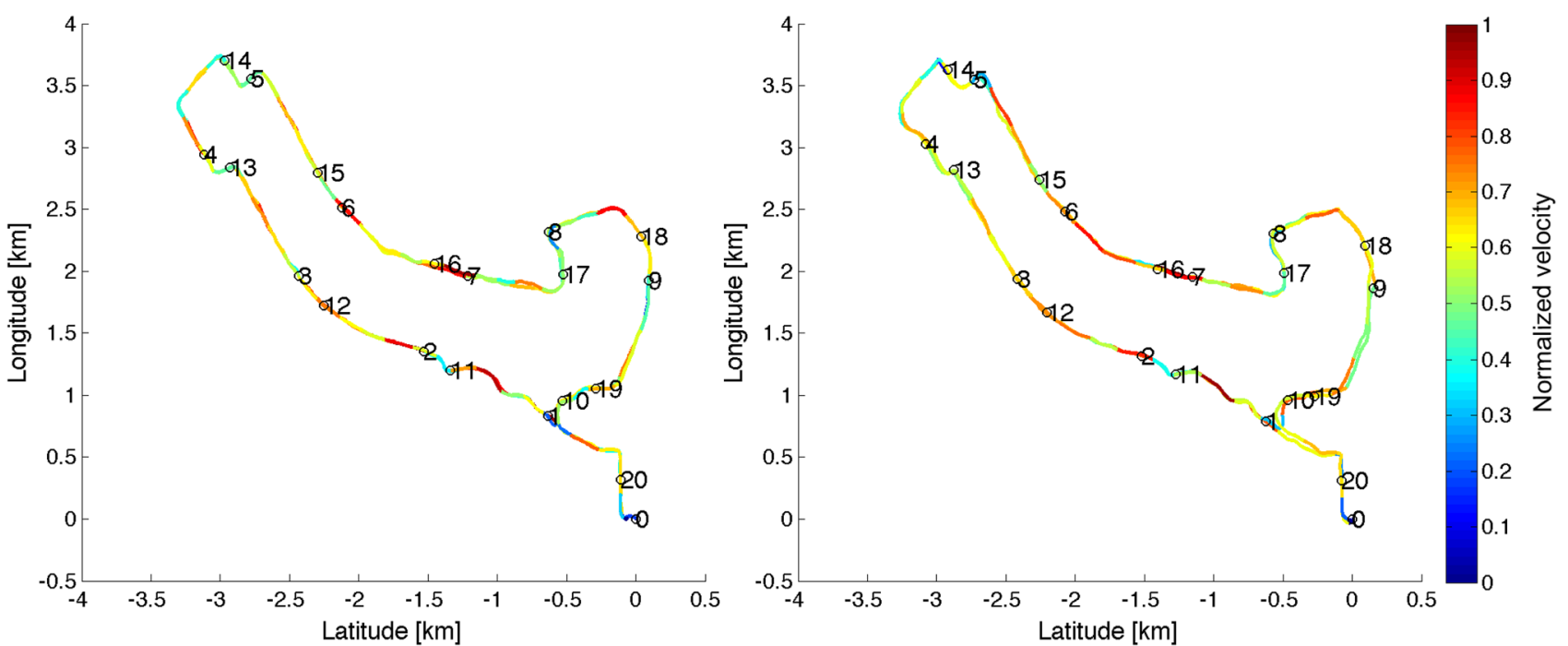

Fig. 4 Remodeled half marathon running track and normalized running velocity examined in $1 \mathrm{~km}$ intervals (running velocity was normalized to the fastest interval for each subject independently) for a middle-aged (a) and younger (b) adult long-distance runner

however no differences in current weekly training volume were found (middle-aged: $47 \mathrm{~km}$ per week vs. younger: $46 \mathrm{~km}$ per week).

\section{Discussion}

The main aim of this study was to examine whether there are different changes in the AT force-elongation relationship between young and middle-aged adult long-distance runners following a half marathon run that might indicate tendon fatigue is age dependent. We hypothesized that the AT would exhibit greater mechanical changes (reduced tendon stiffness), which would be consistent with tendon fatigue, in the middle-aged runners following a half marathon run in comparison to the younger runners.

Our results partly support this hypothesis, showing an increased tendon length at a given force for the middleaged runners immediately after the run. Furthermore, the middle-aged runners showed clear changes in AT stiffness after the run, with an average decrease in tendon stiffness of about $22 \%$. In contrast to this, the younger runners did not show any changes in tendon stiffness, which is in line with previous reports showing no reduction in AT stiffness in vivo following a long-distance run in young adults (Farris et al. 2012; Lichtwark et al. 2013; Peltonen et al. 2012). This was despite a comparable reduction in maximal force production capacity of the younger runners (on average 


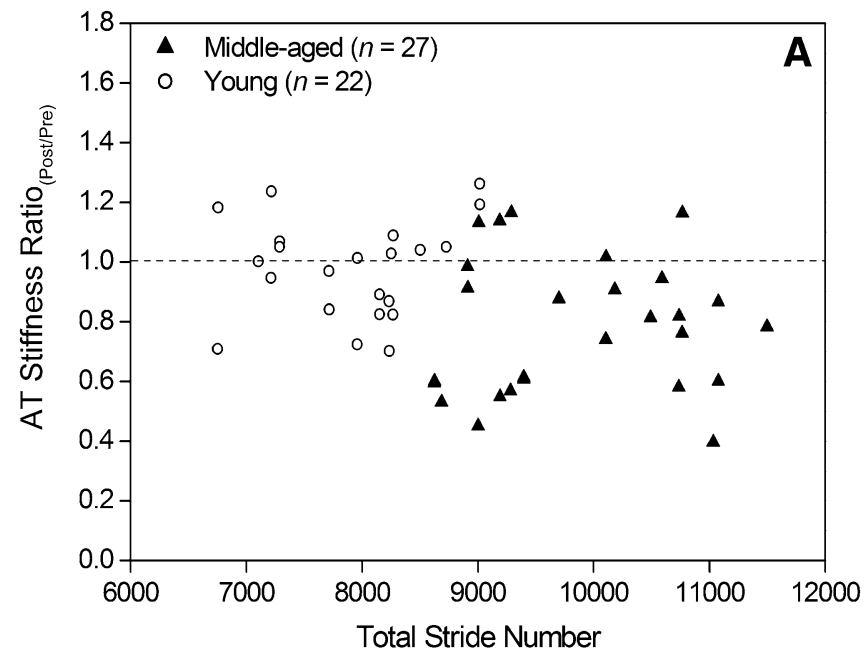

Fig. 5 Relationship between the total number of strides and average stride frequency during the half marathon run and the ratio of tendon stiffness immediately after the run to $20-28 \mathrm{~h}$ before the run for the

$11 \%$ reduction) compared to the middle-aged runners (17\% reduction). The younger runners showed a change in tendon length immediately after the run in the toe region of the force-elongation relationship, up to a force level of $2000 \mathrm{~N}$, compared to the measurements immediately before the run and $20-28 \mathrm{~h}$ after the run. Only some slight tendencies $(0.09<P<0.12)$ for greater elongation values immediately after the run were found at forces between 500 and $1500 \mathrm{~N}$, when compared with the measurement 20-28 $\mathrm{h}$ before the run. This change in elongation could be an indication of a creep response after the $21 \mathrm{~km}$ run, which would be in agreement with the studies of Lichtwark et al. (2013) and Obst et al. (2016). However, in the current study we exclude the fact that in the toe region, any physical damage to the tendon occurred which could be interpreted as tendon fatigue. This creep response was only visible up to $29 \%$ of the maximal force production capacity and was thus not detected in the linear portion of the force-elongation relationship. Therefore, these changes did not influence the tendon stiffness calculated at the higher regions where we did find tendon fatigue for the middleaged subjects. Thus, the above findings suggest that in vivo ATs of older individuals are less capable of resisting longlasting cyclic mechanical loading, induced by long-distance running, altering their tendon mechanical properties in comparison to younger runners.

In the literature, it is well established that during cyclic mechanical loading at a given stress, tendon strain continuously increases and tendon stiffness decreases, with a much faster respective increase and decrease just before rupture (Beaumont 1989; Fung et al. 2010; Wang et al. 1995). We suggest that in the current study, AT fatigue changes

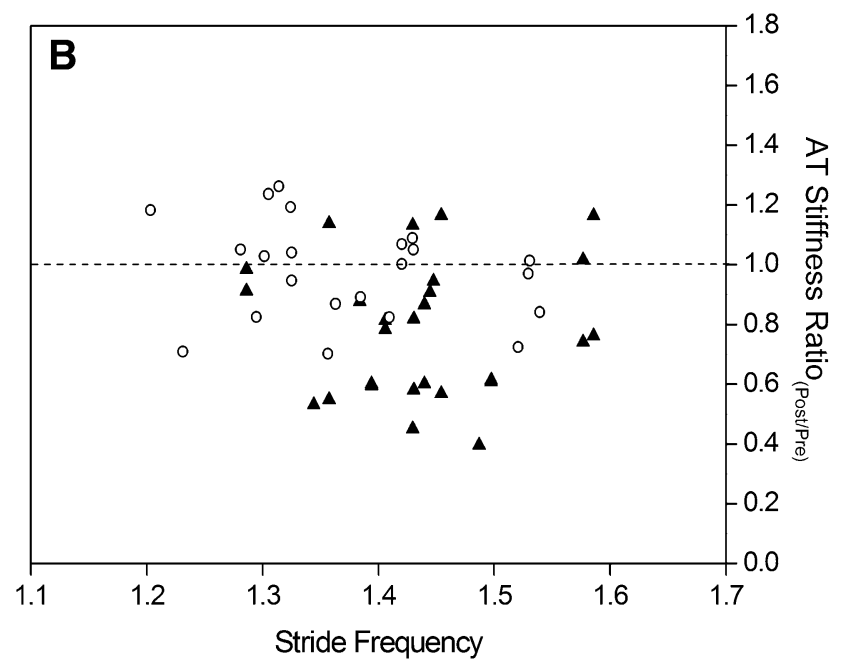

middle-aged (a) and younger (b) adult long-distance runners. No significant effect of stride number and frequency on tendon stiffness ratio was found $\left(R^{2} \leq 0.038, P \geq 0.20\right)$

occurred in the middle-aged, but not in the younger runners. Though the current younger runners demonstrated a decrease in the maximal force production capacity after a half marathon run consisting of on average 7890 AT loading cycles on the examined leg (indicated by the total number of strides), no change in tendon stiffness was seen for the younger runners. The middle-aged runners completed the run using a higher total number of strides and an increased stride frequency compared to the younger runners (old: $9977 \pm 674$ strides vs. young: $7890 \pm 1100$ strides; $1.44 \pm 0.08$ vs. $1.39 \pm 0.10$ strides $\mathrm{s}^{-1}$ ). Thus, one might argue that our observed age-related differences in the AT force-elongation relationship might be related to differences in the frequency and amount of cyclic tensile loading cycles on the tendon between age groups. However, we did not find a significant effect of stride number and frequency on the ratio of the tendon stiffness imPost-Run to 1dayPrerun (Fig. 5). Therefore, it appears that the current changes in tendon properties were related to internal changes within the tendon, rather than stride pattern differences between the age groups.

Although not statistically significant, we cannot exclude the possibility that our observed fatigue changes in tendon stiffness were partly related to slight difference in resting length post-running $(4.7 \mathrm{~mm}$ difference between imPre and imPost for the middle-aged runners). However, because the average reduction in tendon stiffness after the half marathon run in the middle-aged adults was reasonably high (on average $22 \%$ ), this cannot be fully explained by potential changes in tendon resting length post-running. Moreover, to account for a potential effect of the slight difference in resting length on AT mechanical properties, we additionally 
calculated the normalized tendon stiffness (examining of the slope of the force-strain, instead of the force-elongation relationship) and our main findings did not change. A possible explanation for the tendon fatigue found in the middleaged, but not the young, runners may be alterations in viscoelastic and cellular properties in aging tendons, such as a decrease in cell density and altered cell shape (Lavagnino et al. 2013). These alterations have been shown to diminish the cellular contraction rate and, thus, the ability of elongated tendons to re-establish normal tensional homeostasis (Lavagnino et al. 2013). Such age-related changes may be predisposing factors for overuse injuries of tendons (Kaux et al. 2011) and, thus, might lead to tendon fatigue changes.

Despite the tendon stiffness of the middle-aged runners decreasing on average by $22 \%$ immediately after the run, the fatigue changes dissipated $20-28 \mathrm{~h}$ after the exercise. The middle-aged runners showed a tendon stiffness ratio of 0.98 for the measurements $20-28 \mathrm{~h}$ after the run to 20-28 $\mathrm{h}$ before the run. In other words, the results of the current study show that the tendons of middle-aged adults are capable of recovering from acute changes in tendon length-tendon properties within $20-28 \mathrm{~h}$ after a half marathon run. Due to this fast recovery, we suggest that the tendons of the middle-aged runners in the present study did not reach a critical level of AT fatigue at the micro- or nano-structure level. The recovery of our observed changes in the AT force-elongation relationship might be driven by cell-based tendon contraction and viscoelastic recovery, with expected short-term (minutes) and long-term (hours) components (Dahners et al. 1986; Gardner et al. 2012; Lavagnino et al. 2014). Although these recovery mechanisms are negatively affected by age (Bjornsson et al. 2011), they might have played a role in the fast recovery of the tendon within $24 \mathrm{~h}$. Future studies are needed to support the existence of these suggested changes and recovery mechanisms in tendons in vivo.

Regarding our method for assessing the AT mechanical properties in vivo, a number of points should be highlighted. One might argue that a $90^{\circ}$ knee joint angle (instead of full extension), could lead to the GM tendon being tensed at a different starting length, leading to tendon slack (Reeves et al. 2005). However, this measurement error is likely to be small in our stiffness calculation, as we measure the slopes in the linear region (between 30 and $80 \%$ of maximal force) of the force-elongation relationship. Additionally, it could be argued that by using a $90^{\circ}$ knee joint angle, the contribution of the GM muscle to the resultant ankle joint moment during MVC is reduced (Arampatzis et al. 2006), since the GM muscle operates on the ascending limb of the force-length relationship. This drawback may affect our generated tendon length-tension properties. However, it is important to note that using this configuration, we could more tightly fix the limbs in the dynamometer and reduce the soft tissue deformation, which decreases the inevitable ankle joint angular rotation during contraction in comparison to a fully extended knee joint (current study: less than $5^{\circ}$ ankle joint changes during MVC; extended knee: average changes of $14^{\circ}-18^{\circ}$ at the ankle joint; see e.g.: Arampatzis et al. 2005; Karamanidis et al. 2005). Accordingly, we found similar maximal tendon strain values (around $4.5 \%$ at baseline for the young adults), measured at the GM myotendinous junction during MVC, compared to reported GM tendon strain values from fully extended knee joints (Karamanidis and Arampatzis 2005; Mademli and Arampatzis 2008; Mademli et al. 2006; Stenroth et al. 2012). Further, it is mandatory to address the fact that, in the current measurement setup, we estimated the AT force, but examined tendon displacement via ultrasound at the myotendinous junction of the GM, instead of the free AT, affecting our tendon stiffness values in absolute terms. This drawback and the fact that different parts of the tendon and/or aponeurosis show different mechanical properties are, potentially, the reasons that the strain measured at the free AT is approximately two to three times higher compared to the gastrocnemii tendon, at a given ankle joint torque or AT force levels (Farris et al. 2013; Lichtwark et al. 2013; Obst et al. 2016). Therefore, the triceps surae tendon and aponeurosis cannot be considered a single tendon that extends uniformly during plantarflexor contraction. However, when we assessed tendon stiffness in a more dorsiflexed position (ankle joint angle of $85^{\circ}$ ) and thereby increased the force potential of the plantarflexor muscles due to a rightwards shift in the force-length relationship of the contractile element of the gastrocnemii (and soleus muscle), we found no significant differences in tendon stiffness between the two different ankle joint angles (see additional data in Online Resource 1). In addition, it has to be pointed out that while analyzing the more distal myotendinous junction of the soleus by ultrasound seems to be possible in most young adults, it is particularly problematic and in some cases even impossible to detect and analyze the displacement of myotendinous junction of the soleus in many middle-aged or older individuals during contraction. However, to actually examine if different heads of the triceps surae show differences in elongation after a half marathon run between age groups, future studies are needed, possibly examining the influence of a fatiguing protocol on the free AT in middle-aged or older adults. Using the current inverse dynamics approach to calculate AT forces during plantarflexion contraction, we were not able to consider muscle activation and, therefore, we could not account for the contribution of all the other co-activating ankle plantarflexors and antagonist dorsiflexors in our joint kinetic analysis. It is presently difficult to accurately assess in vivo tendon force noninvasively, and without concomitant estimation of in vivo tendon force we can only speculate on the 
significance of the observed age-related findings in tendon mechanical properties. However, we believe that this drawback potentially affects our results in absolute terms more than the validity of the corresponding comparative data. Finally, the AT moment arms were taken from the literature (Maganaris et al. 1998), which might lead to erroneous results in the individual calculation in tendon force and hence tendon stiffness. While this drawback could potentially influence our comparison between middle-aged and younger runners, the main focus was the intraindividual comparison before and after the run and this may not significantly affect the main findings of the current study.

In conclusion, our results suggest that the ATs of middle-aged adult long-distance runners are less capable of resisting prolonged cyclic mechanical loading, significantly altering their tendon force-elongation relationship (increase of tendon elongation and decrease in AT stiffness). However, the fatigue changes observed in the tendon of the middle-aged adult long-distance runners in this study dissipated within 20-28 h, demonstrating the ability of the human tendon in vivo to rapidly recover, which might indicate the existence of both a short-term viscoelastic recovery mechanism and a cell-mediated recovery mechanism in tendons in vivo. Further investigations are necessary to examine the plasticity of human AT in response to short-term mechanical loading in the elderly and which recovery mechanisms are involved in this relatively fast dissipation of the observed fatigue changes of the tendon. Such knowledge may, in the future, contribute to the reduction of AT injuries, especially in middle-aged to older age groups.

Acknowledgments The Bundesinstitut für Sportwissenschaft (BISp), the Olympiastützpunkt Rheinland and the Forschungsservicestelle of the German Sport University Cologne (Hochschulinterne Forschungsförderung) provided the funding for this study. In addition, we would like to thank Dr. Falk Schade for his support throughout this research project.

\section{Compliance with ethical standards}

Conflict of interest The authors declare that they have no conflict of interest.

\section{References}

Arampatzis A, Stafilidis S, DeMonte G, Karamanidis K, MoreyKlapsing G, Brüggemann GP (2005) Strain and elongation of the human gastrocnemius tendon and aponeurosis during maximal plantarflexion effort. J Biomech 38:833-841

Arampatzis A, Karamanidis K, Stafilidis S, Morey-Klapsing G, DeMonte G, Brüggemann GP (2006) Effect of different ankleand knee-joint positions on gastrocnemius medialis fascicle length and EMG activity during isometric plantar flexion. J Biomech 39:1891-1902
Beaumont PWR (1989) The failure of fibre composites: an overview. J Strain Anal Eng 24:189-205

Biewener AA, Roberts TJ (2000) Muscle and tendon contributions to force, work, and elastic energy savings: a comparative perspective. Exerc Sport Sci Rev 28:99-107

Bjornsson HC, Norlin R, Johansson K, Adolfsson LE (2011) The influence of age, delay of repair, and tendon involvement in acute rotator cuff tears: structural and clinical outcomes after repair of 42 shoulders. Acta Orthop 82:187-192

Brunet ME, Cook SD, Brinker MR, Dickinson JA (1990) A survey of running injuries in 1505 competitive and recreational runners. J Sport Med Phys Fit 30:307-315

Clement DB, Taunton JE, Smart GW (1984) Achilles tendinitis and peritendinitis: etiology and treatment. Am J Sport Med 12:179-184

Dahners LE, Banes AJ, Burridge KW (1986) The relationship of actin to ligament contraction. Clin Orthop Relat Res 210:246-251

Farris DJ, Trewartha G, McGuigan MP (2012) The effects of a 30-min run on the mechanics of the human Achilles tendon. Eur J Appl Physiol 112:653-660

Farris DJ, Trewartha G, McGuigan MP, Lichtwark GA (2013) Differential strain patterns of the human Achilles tendon determined in vivo with freehand three-dimensional ultrasound imaging. $\mathbf{J}$ Exp Biol 216:594-600

Fung DT, Wang VM, Andarawis-Puri N et al (2010) Early response to tendon fatigue damage accumulation in a novel in vivo model. J Biomech 43:274-279

Gardner K, Lavagnino M, Egerbacher M, Arnoczky SP (2012) Reestablishment of cytoskeletal tensional homeostasis in lax tendons occurs through an actin mediated cellular contraction of the extracellular matrix. J Orthop Res 30:1695-1701

Huttunen TT, Kannus P, Rolf C, Felländer-Tsai L, Mattila VM (2014) Acute achilles tendon ruptures incidence of injury and surgery in Sweden between 2001 and 2012. Am J Sports Med 42:2419-2423

Jozsa L, Kvist M, Bálint BJ et al (1989) The role of recreational sport activity in Achilles tendon rupture. A clinical, pathoanatomical, and sociological study of 292 cases. Am J Sports Med 17:338-343

Karamanidis K, Arampatzis A (2005) Mechanical and morphological properties of different muscle-tendon units in the lower extremity and running mechanics: effect of aging and physical activity. J Exp Biol 208:3907-3923

Karamanidis K, Stafilidis S, DeMonte G, Morey-Klapsing G, Brüggemann GP, Arampatzis A (2005) Inevitable joint angular rotation affects muscle architecture during isometric contraction. J Electromyogr Kines 15:608-616

Kaux JF, Forthomme B, Le Goff C, Crieland JM, Croisier JL (2011) Current opinions on tendinopathy. J Sports Sci Med 10:238-253

Ker RF, Bennett MB, Bibby SR, Kester RC, Alexander RM (1987) The spring in the arch of the human foot. Nature 325:147-149

Ker RF, Wang XT, Pike AV (2000) Fatigue quality of mammalian tendons. J Exp Biol 203:1317-1327

Kvist M (1994) Achilles tendon injuries in athletes. Sports Med 18:173-201

Lavagnino M, Gardner K, Arnoczky SP (2013) Age-related changes in the cellular, mechanical, and contractile properties of rat tail tendons. Connect Tissue Res 54:70-75

Lavagnino M, Bedi A, Walsh CP, Enselman ERS, Sheibani-Rad S, Arnoczky SP (2014) Tendon contraction after cyclic elongation is an age-dependent phenomenon in vitro and in vivo comparisons. Am J Sport Med 42:1471-1477

Lichtwark GA, Bougoulias K, Wilson AM (2007) Muscle fascicle and series elastic element length changes along the length of the human gastrocnemius during walking and running. J Biomech 40:157-164 
Lichtwark GA, Cresswell AG, Newsham-West RJ (2013) Effects of running on human Achilles tendon length-tension properties in the free and gastrocnemius components. J Exp Biol 216:4388-4394

Mademli L, Arampatzis A (2008) Mechanical and morphological properties of the triceps surae muscle-tendon unit in old and young adults and their interaction with a submaximal fatiguing contraction. J Electromyogr Kines 18:89-98

Mademli L, Arampatzis A, Walsh M (2006) Effect of muscle fatigue on the compliance of the gastrocnemius medialis tendon and aponeurosis. J Biomech 39:426-434

Maganaris CN (2003) Tendon conditioning: artefact or property? Proc Biol Sci 270(Suppl 1):S39-S42

Maganaris CN, Baltzopoulos V, Sargeant AJ (1998) Changes in Achilles tendon moment arm from rest to maximum isometric plantarflexion: in vivo observations in man. J Physiol 510:977-985

McCamley J, Donati M, Grimpampi E, Mazza C (2012) An enhanced estimate of initial contact and final contact instants of time using lower trunk inertial sensor data. Gait Posture 36:316-318

Noyes FR, Grood ES (1976) The strength of the anterior cruciate ligament in humans and Rhesus monkeys. J Bone Joint Surg 58:1074-1082

Obst SJ, Newsham-West R, Barrett RS (2016) Changes in Achilles tendon mechanical properties following eccentric heel drop exercise are specific to the free tendon. Scand J Med Sci Spor $26: 421-431$

Peltonen J, Cronin NJ, Avela J, Finni T (2010) In vivo mechanical response of human Achilles tendon to a single bout of hopping exercise. J Exp Biol 213:1259-1265

Peltonen J, Cronin NJ, Stenroth L, Finni T, Avela J (2012) Achilles tendon stiffness is unchanged $1 \mathrm{~h}$ after a marathon. J Exp Biol 215:3665-3671

Reeves ND, Maganaris CN, Ferretti G, Narici MV (2005) Influence of 90-day simulated microgravity on human tendon mechanical properties and the effect of resistive countermeasures. J Appl Physiol 98:2278-2286

Stenroth L, Peltonen J, Cronin NJ, Sipilä S, Finni T (2012) Agerelated differences in Achilles tendon properties and triceps surae muscle architecture in vivo. J Appl Physiol 113:1537-1544

Tkaczuk H (1968) Tensile properties of human lumbar longitudinal ligaments. Acta Orthop Scand 39(sup115):1-69

Viidik A (1982) Age-related changes in connective tissues. In: Viidik A (ed) Lectures on gerontology. Academic Press, London, pp 173-211

Wang XT, Ker RF, Alexander RM (1995) Fatigue rupture of wallaby tail tendons. J Exp Biol 198:847-852 